\title{
Article \\ Structural Recognition of Triple-Stranded DNA by Surface-Enhanced Raman Spectroscopy
}

\author{
Luca Guerrini ${ }^{1, *(D)}$ and Ramon A. Alvarez-Puebla ${ }^{1,2, *(D)}$ \\ 1 Department of Physical and Inorganic Chemistry_EMaS, Universitat Rovira I Virgili, Carrer de Marcel·lí \\ Domingo s/n, 43007 Tarragona, Spain \\ 2 ICREA, Passeig Lluís Companys 23, 08010 Barcelona, Spain \\ * Correspondence: luca.guerrini@urv.cat (L.G.); ramon.alvarez@urv.cat (R.A.A.-P.)
}

check for updates

Citation: Guerrini, L.;

Alvarez-Puebla, R.A. Structural Recognition of Triple-Stranded DNA by Surface-Enhanced Raman Spectroscopy. Nanomaterials 2021, 11, 326. https://doi.org/10.3390/ nano11020326

Academic Editor: Andrew Pike Received: 24 December 2020 Accepted: 21 January 2021 Published: 27 January 2021

Publisher's Note: MDPI stays neutral with regard to jurisdictional claims in published maps and institutional affiliations.

Copyright: (C) 2021 by the authors. Licensee MDPI, Basel, Switzerland. This article is an open access article distributed under the terms and conditions of the Creative Commons Attribution (CC BY) license (https:// creativecommons.org/licenses/by/ $4.0 /)$.

\begin{abstract}
Direct, label-free analysis of nucleic acids via surface-enhanced Raman spectroscopy (SERS) has been continuously expanding its range of applications as an intriguing and powerful analytical tool for the structural characterization of diverse DNA structures. Still, interrogation of nucleic acid tertiary structures beyond the canonical double helix often remains challenging. In this work, we report for the first time the structural identification of DNA triplex structures. This class of nucleic acids has been attracting great interest because of their intriguing biological functions and pharmacological potential in gene therapy, and the ability for precisely engineering DNA-based functional nanomaterials. Herein, structural discrimination of the triplex structure against its duplex and tertiary strand counterparts is univocally revealed by recognizing key markers bands in the intrinsic SERS fingerprint. These vibrational features are informative of the base stacking, Hoogsteen hydrogen bonding and sugar-phosphate backbone reorganization associated with the triple helix formation. This work expands the applicability of direct SERS to nucleic acids analysis, with potential impact on fields such as sensing, biology and drug design.
\end{abstract}

Keywords: surface-enhanced Raman spectroscopy; DNA; triplex; plasmonic nanoparticles

\section{Introduction}

The polymorphic nature of DNA enables the adoption of a diverse range of structural arrangements beyond the right-handed B-form duplex, including non-canonical structures such as G-quadruplexes, triplexes, and i-motifs [1]. These structures have been shown to participate in multiple biological processes and, remarkably, their forming sequences have been recognized as sources of genetic instability, contributing to human diseases and genetic disorders [1,2].

Among non-canonical conformations, triplex structures have attracted great interest. Besides the understanding of their biological functions, they display pharmacological potential in gene therapy based on their ability to modulate gene expression, such as via inhibition of disease-related genes and generation of site-specific mutations [2,3]. Additionally, triplex nucleic acids have been emerging as a powerful tool in the field of DNA nanotechnology where their ability for structural reconfiguration is exploited for engineering DNA-based functional nanomaterials via a diverse set of external stimuli $[4,5]$.

Structurally speaking, triplex nucleic acids can be classified as intramolecular structures that are naturally occurring at DNA regions containing homopurine and homopyrimidine segments with mirror repeat symmetry, or intermolecular multistrand assemblies resulting from the binding of a triplex-forming oligonucleotide (TFO) to a target duplex structure $[1,3]$. TFOs can bind double helices with high affinity and sequence specificity via Hoogsteen hydrogen bonds, positioning in the major groove of the duplex. These triplexforming strands consist of pyrimidine or purine-rich sequences that bind their counterparts (a purine or pyrimidine segment) in the double helix in either parallel or anti-parallel direction $[2,3]$. Besides the nucleobase sequence and length, surrounding conditions also 
play a central role in the generation of stable triplex assemblies. These variables include the solution $\mathrm{pH}$ and the presence of divalent cations (e.g., $\mathrm{Mg}^{2+}$ ) or naturally occurring polyamines (e.g., spermine), which can act as triplex stabilizers by reducing the extent of electrostatic repulsion between the phosphate backbones [2-4].

In-depth characterization of the structural, kinetic and thermodynamic properties of triplexes is of utmost importance for the correct understanding of their biological role and to improve their efficiency and specificity in gene therapy [3,4]. Techniques, such as nuclear magnetic resonance (NMR), X-ray fiber diffraction (XRD), and other optical spectroscopies (e.g., circular dichroism, UV-Vis and fluorescence-based methods) have been preferably applied to this purpose $[4,6]$. To a much lesser extent, structural data on triplex structures have been also collected using Raman spectroscopy [7-10]. Raman spectroscopy is a vibrational technique which has been lengthily exploited for the detailed structural characterization of biomolecules, including nucleic acid structures. Importantly, the possibility of performing Raman studies on nucleic acids in their natural state (i.e., in solution, rather than in crystal form such as for XRD) increases the biological significance of the obtained data. However, the Raman scattering phenomenon is a very inefficient process, which drastically limits the sensitivity of this spectroscopy and, thus, hampers its application to molecular systems at low-concentration levels. This issue can be overcome via plasmon-mediated enhancement of the Raman scattering from a molecular entity located near a metallic nanostructured surface when localized surface plasmon resonances (LSPRs) are efficiently excited upon illumination with an appropriate light source [11]. The integration of Raman technique and plasmonics paved the way for the development of a new method termed surface-enhanced Raman spectroscopy (SERS) which preserves the structural specificity, high experimental flexibility and simplicity of the traditional method while imparting extremely high sensitivity. This enabled the application of SERS as an ultrasensitive analytical approach for the structural characterization of (bio)molecular species as well as sensing tool for their detection and quantification [11-13]. However, while the use of SERS in indirect approaches for nucleic acids sensing has been extensively dealt with in the literature, its wider use as an ultrasensitive analytical tool for this class of biomolecules via the acquisition of their intrinsic SERS vibrational fingerprinting (i.e., direct SERS) has been traditionally far less explored, until relatively recently [14-22]. Our group devised a novel approach based on the use of positively charged silver colloids as plasmonic-enhancers which successfully tackled prior issues associated with the reproducible acquisition of reliable SERS spectra of nucleic acids at low concentrations $[19,23,24]$. Notably, the use of this class of silver nanoparticles affords high sensitivity for discriminating DNA conformations (i.e., singlevs. double-stranded helix) which is otherwise poor for traditional negatively charged colloids [25].

To the best of our knowledge, only two articles from 1996 reported the acquisition of triplex SERS spectra, specifically from RNA triple helices adsorbed on silver electrodes [26, 27]. However, the illustrated SERS data display spectral profiles with no actual resemblance to the characteristic vibrational patterns of nucleic acids. This incongruence was later elucidated by noting the striking correspondence of these anomalous spectra to the SERS of methylviologen, a molecule commonly used in electrochemistry such as for electrode roughening, which has been indicated as the actual source of the intense signal [28].

Herein, we report thus the first unequivocal recognition of a DNA triplex structure by SERS spectroscopy. Specifically, we investigated an intermolecular triple-helix comprising a 31-base pair duplex bound to a 22-nucleotide pyrimidine-rich single strand. The molecular fingerprint information contained in the SERS spectra allowed for the structural discrimination and characterization of the free single strand and the same sequence involved in the triplex construct. This study expands the applicability of SERS spectroscopy as a powerful molecular tool for structural interrogation of nucleic acids to the intriguing class of triplex structures, with potential impact on fields such as sensing, biology, drug-design and DNA nanotechnology. 


\section{Materials and Methods}

\subsection{Materials}

All chemicals were obtained from Sigma-Aldrich and Fisher Scientific at the highest purity available. Lyophilized oligonucleotides were purchased from Eurofins and dissolved in milli-Q water to approximately $300 \mu \mathrm{M}$ concentration. The exact DNA concentrations were then estimated by measuring the absorbance at $260 \mathrm{~nm}$. Subsequently, DNA samples were diluted to $40 \mu \mathrm{M}$ in triplex buffer (sodium-magnesium phosphate buffer solution $\mathrm{pH} 7,120 \mathrm{mM} \mathrm{NaCl}, 8 \mathrm{mM} \mathrm{Mg}^{2+}$ ). Duplex formation was achieved by heating to $90{ }^{\circ} \mathrm{C}$ for $10 \mathrm{~min}$ an equimolar solution $(20 \mu \mathrm{M})$ of complementary strands in triplex buffer. The solution was then slowly cooled down to room temperature. All DNA solutions were kept at $4{ }^{\circ} \mathrm{C}$ before their experimental use. Triplex formation was performed by adding an equimolar amount of the triplex-forming oligonucleotide (TFO) to the duplex nucleic acid in triplex buffer. The mixture was allowed to equilibrate at $4{ }^{\circ} \mathrm{C}$ for $24 \mathrm{~h}$.

\subsection{Synthesis of Positively Charged Silver Colloids and SERS Measurements}

Synthesis of positively charged spermine coated-silver nanoparticles (AgSp) was carried out as previously reported [29,30]. Briefly, to $10 \mathrm{~mL}$ of Milli-Q water were consecutively added, under stirring, $20 \mu \mathrm{L}$ of silver nitrate $0.5 \mathrm{M}$ and $7 \mu \mathrm{L}$ of spermine tetrahydrochloride $0.1 \mathrm{M}$ solutions. After $20 \mathrm{~min}, 250 \mu \mathrm{L}$ of sodium borohydride $0.01 \mathrm{M}$ were quickly added to the mixture under vigorous stirring. The colloids were then left to settle overnight and the deposit at the bottom of the vial was discarded. To prevent the adhesion of AgSp nanoparticles onto glass surfaces, vials were previously coated with polyethyleneimine. Samples for SERS experiments were prepared as follows. All DNA samples were previously diluted to $20 \mu \mathrm{M}$ with their corresponding solvent (milli-Q water or triplex buffer). Then, $0.9 \mu \mathrm{L}$ of these solutions were added to $150 \mu \mathrm{L}$ of colloids (stored at $4{ }^{\circ} \mathrm{C}$ ), and quickly stirred. Samples were left to equilibrate for at least $3 \mathrm{~h}$ at $4{ }^{\circ} \mathrm{C}$, and briefly sonicated before the acquisition of the SERS spectra.

\subsection{Instrumentation}

Scanning UV-vis spectra were obtained using a Thermo Scientific Evolution 201 UVvisible spectrophotometer. Transmission electron microscopy, TEM, images were collected using a JEOL 1011 (JEOL USA, Inc, Peabody, MA) operating at $100 \mathrm{kV}$. TEM samples were prepared by diluting the sample before their deposition onto carbon-Formvar-coated 200 mesh copper grids. SERS spectra were obtained using a Renishaw InVia Reflex confocal microscope equipped with a $514 \mathrm{~nm}$ laser. A lens for macrosampling (30 $\mathrm{mm}$ focal length, $0.17 \mathrm{NA}$ ) was employed to focus the laser onto the sample. The averaged SERS spectra illustrated in the manuscript were typically obtained under the following conditions: $10 \mathrm{~s}$ exposure time, 15 accumulations, $100 \%$ laser power.

\section{Results and Discussion}

The plasmonic substrate for SERS characterization of nucleic acids consists of colloidal spermine-coated silver nanoparticles (AgSp) with a narrow LSPR centered at ca. $390 \mathrm{~nm}$ (Figure 1A) and an average diameter of $20.0 \pm 4.1 \mathrm{~nm}$ (Figure 1B, inset and Figure S1A). Polyaminic spermine molecules adsorbed onto the silver surface impart an overall positive charge to the silver nanostructures $(\zeta$-potential $=+40 \mathrm{mV})$. The addition of tiny amounts of triplex buffer solution (sodium-magnesium phosphate buffer solution $\mathrm{pH} 7,120 \mathrm{mM}$ $\mathrm{NaCl}, 8 \mathrm{mM} \mathrm{Mg}^{2+}$ ) induces a relatively slow but unrestrained nanoparticle aggregation (Figure 1A). On the other hand, the resulting SERS spectrum (Figure 1B) shows a low background signal in the spectral region of interest for DNA analysis (ca. $400-1800 \mathrm{~cm}^{-1}$ ). 

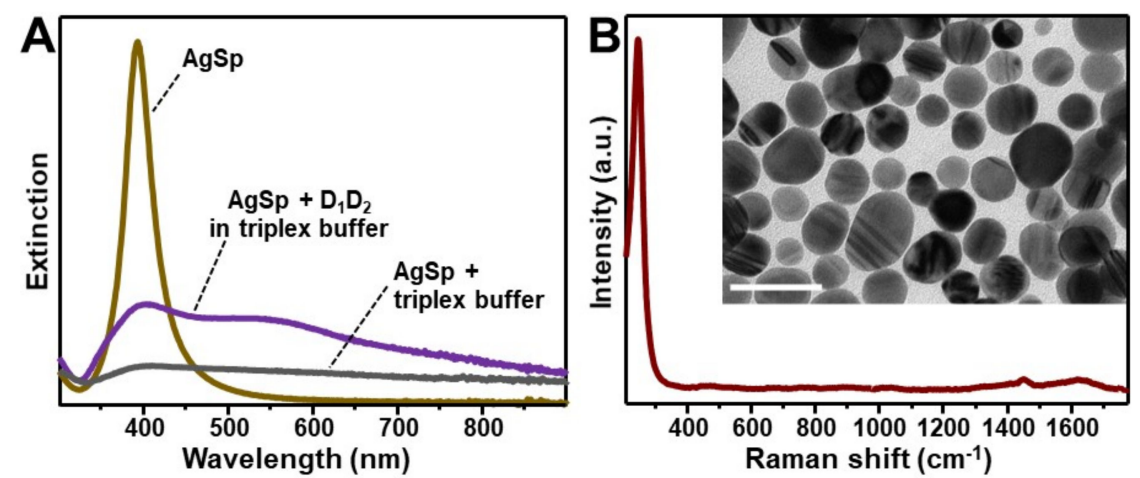

Figure 1. (A) Extinction spectrum of spermine-coated silver colloids (AgSp, $150 \mu \mathrm{L}$ ) before and $3 \mathrm{~h}$ after the addition of $0.9 \mu \mathrm{L}$ of (a) triplex buffer (grey line) or (b) a $20 \mu \mathrm{M}$ solution of $\mathrm{D}_{1} \mathrm{D}_{2}$ duplex in triplex buffer (purple line). (B) Surface-enhanced Raman spectroscopy (SERS) spectrum of AgSp upon addition of $0.9 \mu \mathrm{L}$ of triplex buffer. The intense band at $244 \mathrm{~cm}^{-1}$ is ascribed to the $v(\mathrm{Ag}-\mathrm{Cl})$ vibration. Inset: representative transmission electron microscopy (TEM) image of the nanoparticles (scale bar: $50 \mathrm{~nm}$ ).

As a model intermolecular triple-helix (Figure 2A), we selected one based on the association of a 31-base pair duplex $\left(\mathrm{D}_{1} \mathrm{D}_{2}\right)$, comprising a pyrimidine-rich strand $\left(\mathrm{D}_{1}\right)$ and a purine-rich strand $\left(\mathrm{D}_{2}\right)$, with a 22-nucleotide pyrimidine-rich strand (TFO). The third strand binds via formation of $19 \mathrm{~T} \bullet \mathrm{AT}$ and $2 \mathrm{C} \bullet \mathrm{GC}$ base triplets adopting a parallel orientation along the major groove of the purine-rich $\mathrm{D}_{2}$ strand in the Watson-Crick $\mathrm{D}_{1} \mathrm{D}_{2}$ duplex [31]. The resulting $\mathrm{D}_{1} \mathrm{D}_{2} \bullet \mathrm{TFO}$ construct (1:1:1 ratio, where "•" indicates the Hoogsteen interactions) has been previously characterized by conventional techniques, demonstrating to possess high stability at room temperature in a pseudo-physiological triplex buffer ( $\mathrm{pH} 7,120 \mathrm{mM} \mathrm{NaCl}, 8 \mathrm{mM} \mathrm{Mg}^{2+}$ ) [31]. The triplex construct was obtained by combining an equimolar amount of TFO and $\mathrm{D}_{1} \mathrm{D}_{2}$ duplex for $24 \mathrm{~h}$ at $4{ }^{\circ} \mathrm{C}$. In fact, while generating very stable structures, triplex formation is kinetically much slower than duplex annealing [2].

Identical volumes of DNA containing triplex buffers of the different samples (i.e., duplex $D_{1} D_{2}$, triplex $D_{1} D_{2} \bullet T F O$, and single-strand TFO) were added to AgSp colloids. Differently to what previously observed (Figure 1A), the nanoparticles undergo rapid aggregation into stable clusters in suspension due to the electrostatic adhesion of the nucleic acids mediated by the phosphate backbone-spermine interactions. Representative transmission electron microscopy (TEM) images of the AgSp colloids upon addition of either triplex buffer or $D_{1} D_{2}$ in triplex buffer (Figure $S 1 B, C$ ) provide complementary qualitative evidence of the different degree of aggregation underwent by the nanoparticles.

Figure 2B shows the corresponding SERS spectra. Typically, difference spectroscopy is applied to both Raman and SERS analysis of nucleic acids to better expose the spectral differences emerging from the complex vibrational patterns of these large biomolecules. To this end, digital subtraction between spectra is carried out using the $\mathrm{VPO}_{2}{ }^{-}$band at ca. $1089 \mathrm{~cm}^{-1}$ as internal standard, due to its large insensitivity to structural alterations [14]. In our specific case, since duplex and triplex structures possess different molar content of phosphate groups (i.e., $84 / \mathrm{mol}$ for $\mathrm{D}_{1} \mathrm{D}_{2} \bullet T F O$, and $62 / \mathrm{mol}$ for $\mathrm{D}_{1} \mathrm{D}_{2}$ ), the intensity of their corresponding SERS spectra was proportionally adjusted to the peak height of the ca. $1089 \mathrm{~cm}^{-1}$ band according to the relative number of $\mathrm{PO}_{2}^{-}$per molecular construct. This allows extracting a difference spectrum $\mathrm{D}_{1} \mathrm{D}_{2} \bullet T F O-\mathrm{D}_{1} \mathrm{D}_{2}$ which primarily represents the vibrational pattern of TFO in the triplex structures and, thus, can be meaningfully compared to the SERS profile of the unbound TFO strand. The SERS spectrum of TFO is dominated by thymine bands [25], as expected from the relative nucleobase content. Still, it can be distinguished a shoulder approximately located at $1630 \mathrm{~cm}^{-1}$ which has been attributed ascribed to $v\left(\mathrm{C}_{2}=\mathrm{O}_{4}\right)$ modes of cytosine. Additionally, based on our previous works $[23,25,29]$, we assume non-negligible contributions from cytosine in-plane ring 
vibrations in the complex ensemble of overlapping features in the $1150-1600 \mathrm{~cm}^{-1}$ range as well as for the ring breathing band at ca. $792 \mathrm{~cm}^{-1}$. Figure 2C-E illustrate the SERS signals of TFO and the digitally subtracted $D_{1} D_{2} \bullet T F O-D_{1} D_{2}$ in three key spectral regions of interest. For the sake of comparison, the intensities of the two spectra were arbitrarily modified to better visualize the discussed spectral changes. The intense feature centered at ca. $792 \mathrm{~cm}^{-1}$ of TFO (Figure 2C, green curve) undergoes a $3 \mathrm{~cm}^{-1}$ blue-shift and a broadening in the $\mathrm{D}_{1} \mathrm{D}_{2} \bullet T F O-\mathrm{D}_{1} \mathrm{D}_{2}$ difference spectrum (Figure $2 \mathrm{C}$, brown curve), which is informative of a perturbation of the nucleobase electronic properties associated with an increase in base stacking. The extent of spectral shift is significantly lower than the one observed upon hybridization of a polythymine strand with a complementary polyadenine sequence to form the corresponding duplex (ca. $7 \mathrm{~cm}^{-1}$ blue-shift) [29], which is consistent with the knowledge that the stacking interactions of TFO nucleobases in triplex constructs are weaker [1]. This spectral change is mirrored by the ca. $4 \mathrm{~cm}^{-1}$ red-shift of the thymine $v\left(\mathrm{C}_{4}=\mathrm{O}_{4}\right)$ band at $1644 \mathrm{~cm}^{-1}$ (Figure 2E), primarily sensitive to base pairing, which suggests the involvement of the carbonyl group in hydrogen bonding (Figure 2A, inset) [25]. Finally, clues of changes in the sugar-phosphate backbone conformation are mostly reflected in the $800-1100 \mathrm{~cm}^{-1}$ spectral range. Here, we observe the clear change in intensity ratio between the vibration of the ribose sugar at ca. $1020 \mathrm{~cm}^{-1}$ and the abovementioned $\nu \mathrm{PO}_{2}{ }^{-}$band at ca. $1089 \mathrm{~cm}^{-1}$. A similar trend was also previously reported for polythymine strands forming double helix structures [29,32]. The set of all spectral changes is therefore consistent with the binding of the TFO to the purine-rich strand in the $\mathrm{D}_{1} \mathrm{D}_{2}$ duplex via Hoogsteen base pairing and stacking interactions with the neighboring bases. It is also worth noting that, while not vibrationally interpreted due to the intricacy of band overlapping, relevant spectral alterations were also detected in the $1200-1450 \mathrm{~cm}^{-1}$ spectral range (Figure S2). The SERS spectra in Figure 2B are the averaged results of three separate replicas, whose original non-baseline spectra are illustrated in Figure S3 (the data show high reproducible spectral profiles with only minimal fluctuations of the broad continuum background).

As an experimental control, we acquired the SERS spectrum of an equimolar mixture of the TFO and a non-complementary 21-bp duplex $\left(\mathrm{dsDNA}_{21}\right)$ and compared to the SERS signal of the duplex alone via digital subtraction (Figure 3 ), as similarly performed for $\mathrm{D}_{1} \mathrm{D}_{2}$ in Figure 2B. In this case, the corresponding difference spectrum $\left(\mathrm{dsDNA} \mathrm{A}_{21}+\right.$ $\mathrm{TFO}-\mathrm{dsDNA}_{21}$ does not reveal any distinguishable vibrational pattern. This result is not surprising considering our previous SERS study on mixtures of a 21-nt single-stranded DNA (ssDNA) and a 21-bp duplex, which showed that the SERS signal is fully dominated by the duplex contribution for single-strand content below $80 \%$ [29]. Such outcome was attributed to a superior ability of DNA duplexes in mediating the formation of highly efficient interparticle hotspots as compared to single-stranded sequences, which may be based on the different availability of negatively charged phosphate groups. Thus, the experimental control further corroborates the validity of the data presented in Figure 2, that is the acquired SERS spectrum of the $\mathrm{D}_{1} \mathrm{D}_{2}+$ TFO equimolar mixture indeed represents the vibrational fingerprint of the intermolecular triplex. 

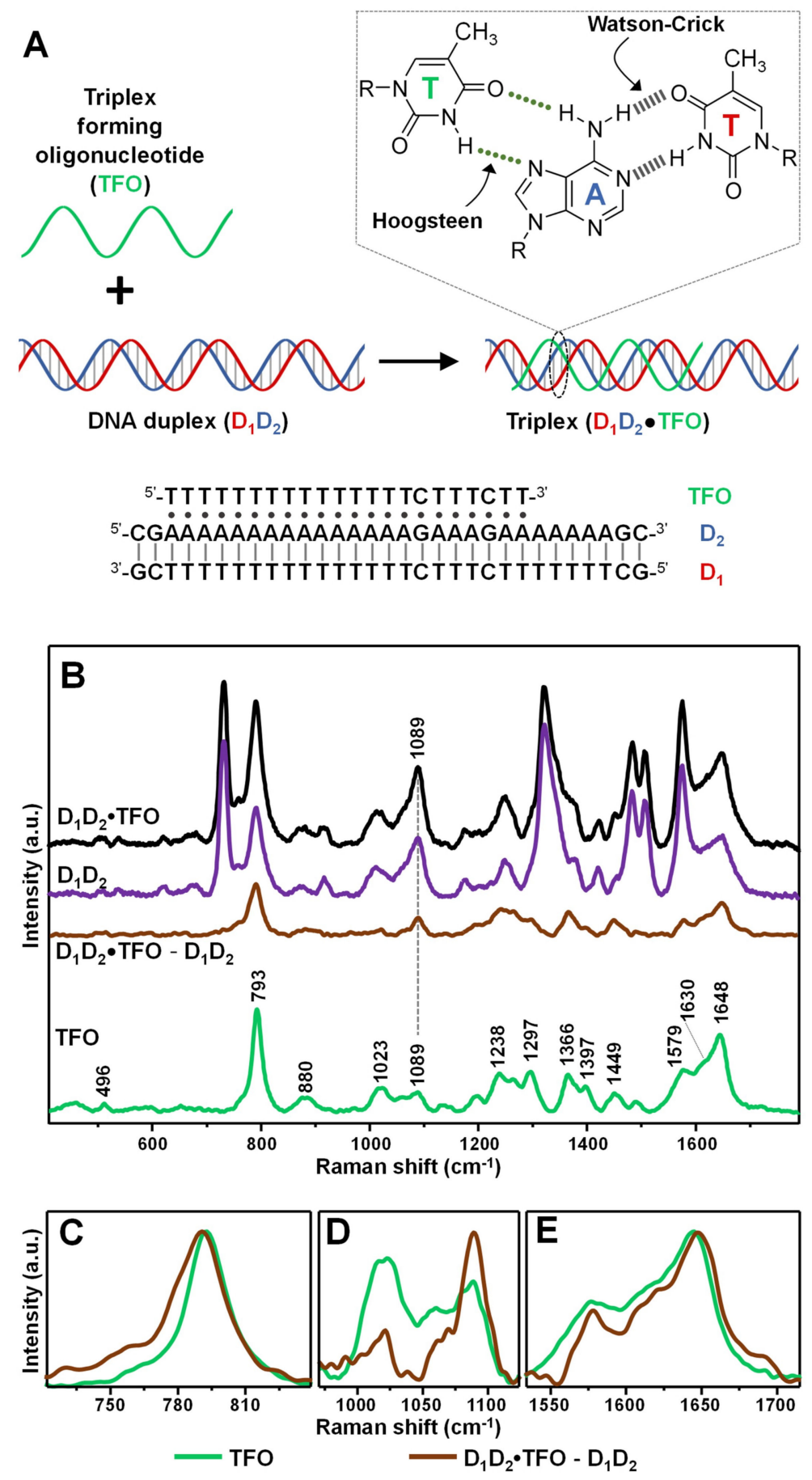

Figure 2. (A) Outline of the intermolecular triplex formation and corresponding oligonucleotide sequences (where "•" and "-" indicates Hoogsteen and Watson-Crick base pairings, respectively). Inset: chemical structure of a parallel T•AT triplet. (B) SERS spectra of the intermolecular triplex $\mathrm{D}_{1} \mathrm{D}_{2} \bullet$ TFO, duplex $\mathrm{D}_{1} \mathrm{D}_{2}$, and the individual TFO. The $\mathrm{D}_{1} \mathrm{D}_{2} \bullet$ TFO and $\mathrm{D}_{1} \mathrm{D}_{2}$ SERS spectra were normalized to the intensity of the $\mathrm{VPO}_{2}^{-}$band at $1089 \mathrm{~cm}^{-1}$ according to the relative content of phosphate groups in the construct (i.e., 84 for $\mathrm{D}_{1} \mathrm{D}_{2} \bullet T F O$, and 62 for $\mathrm{D}_{1} \mathrm{D}_{2}$ ). $\mathrm{D}_{1} \mathrm{D}_{2} \bullet T F O-T F O$ is the difference SERS spectrum obtained upon digital subtraction of the duplex spectrum to the triplex spectrum. (C-E) Details of the 720-840, 960-1180 and 1530-1715 $\mathrm{cm}^{-1}$ spectral regions, respectively, for TFO spectrum (green curve) and difference $\mathrm{D}_{1} \mathrm{D}_{2} \bullet \mathrm{TFO}-\mathrm{TFO}$ spectrum (brown curve). For the sake of a better comparison, the intensities of the spectra were arbitrarily modified in each of these three figures. 


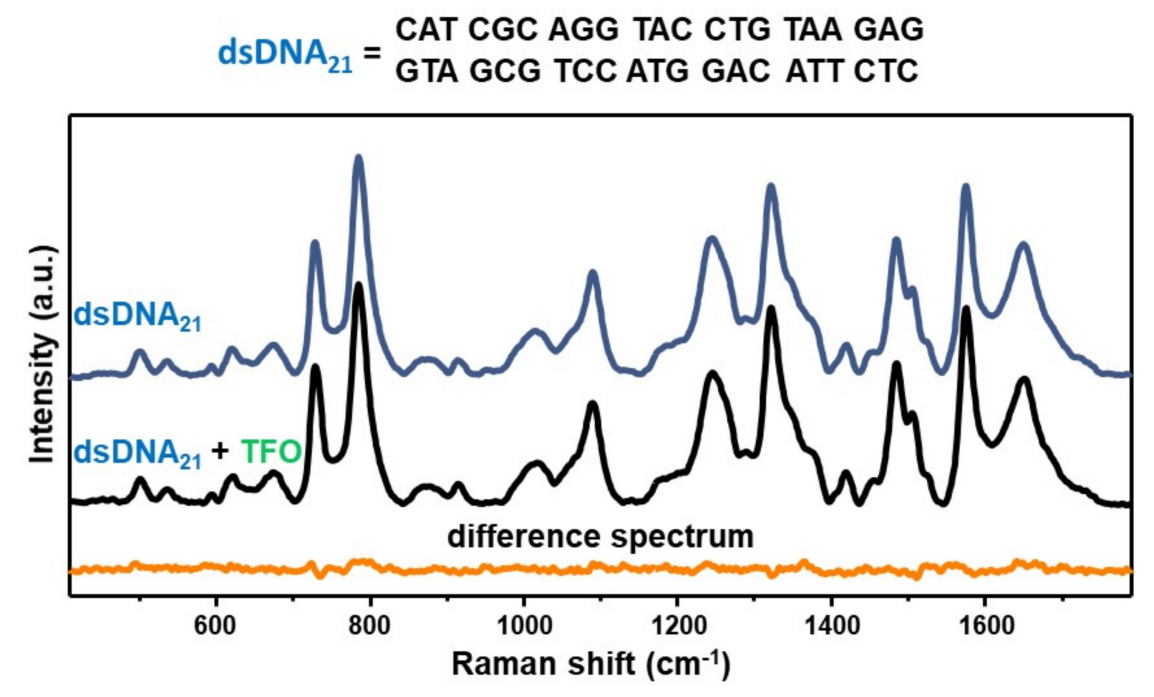

Figure 3. SERS spectra of the 21-base pairs duplex dsDNA 21 (blue curve) and the equimolar mixture $\mathrm{dsDNA}_{21}+\mathrm{TFO}$ (black curve). Orange curve: difference spectrum (dsDNA $\left.21+\mathrm{TFO}\right)-\mathrm{dsDNA}_{21}$ obtained by digitally subtracting the blue curve to the black one.

In summary, structural discrimination of the triplex structure against its duplex and tertiary strand counterparts is unambiguously revealed by recognizing key markers bands in their intrinsic SERS fingerprints. These vibrational features are informative of the base stacking, Hoogsteen hydrogen bonding and sugar-phosphate backbone reorganization associated with the triple helix formation. This work paves the way for the application of direct, label-free analysis of nucleic acids via SERS spectroscopy beyond the single strand DNAs and canonical double helix forms to this intriguing class of tertiary structures which captivated the attention due to, among others, their biological functions and pharmacological potential in gene therapy.

Supplementary Materials: The following are available online at https:/ / www.mdpi.com/2079-499 1/11/2/326/s1, Figure S1: size histogram of the silver nanoparticles, TEM images of the colloids upon addition of triplex buffer or $\mathrm{D}_{1} \mathrm{D}_{2}$ in triplex buffer. Figure S2: SERS spectrum of TFO and difference $\left(D_{1} D_{2} \bullet\right.$ TFO- $\left.D_{1} D_{2}\right)$ spectrum in the $450-1750 \mathrm{~cm}^{-1}$ range. Figure S3: SERS spectra of $\mathrm{TFO}, \mathrm{D}_{1} \mathrm{D}_{2} \bullet \mathrm{TFO}$ and $\mathrm{D}_{1} \mathrm{D}_{2}$ from different replicas.

Author Contributions: L.G. carried out the experiments. L.G. and R.A.A.-P. conceived and designed the experiments, analyzed the data, and wrote the manuscript. All authors have read and agreed to the published version of the manuscript.

Funding: This work was funded by the Spanish Ministerio de Economía, Industria y Competitividad (CTQ2017-88648R, RYC-2016-20331), the Generalitat de Cataluña (2017SGR883), the Universitat Rovira i Virgili (2018PFRURV-B2-02), and the Universitat Rovira i Virgili and Banco Santander (2017EXIT-08).

Institutional Review Board Statement: Not applicable.

Informed Consent Statement: Not applicable.

Data Availability Statement: The data presented in this study are available on request from the corresponding authors.

Conflicts of Interest: The authors declare no conflict of interest.

\section{References}

1. Tateishi-Karimata, H.; Sugimoto, N. Chemical biology of non-canonical structures of nucleic acids for therapeutic applications. Chem. Commun. 2020, 56, 2379-2390. [CrossRef] [PubMed]

2. Jain, A.; Wang, G.; Vasquez, K.M. DNA triple helices: Biological consequences and therapeutic potential. Biochimie 2008, 90, 1117-1130. [CrossRef] [PubMed] 
3. Chan, P.P.; Glazer, P.M. Triplex DNA: Fundamentals, advances, and potential applications for gene therapy. J. Mol. Med. 1997, 75, 267-282. [CrossRef] [PubMed]

4. Hu, Y.; Cecconello, A.; Idili, A.; Ricci, F.; Willner, I. Triplex DNA nanostructures: From basic properties to applications. Angew. Chem. Int. Edit. 2017, 56, 15210-15233. [CrossRef]

5. Chandrasekaran, A.R.; Rusling, D.A. Triplex-forming oligonucleotides: A third strand for DNA nanotechnology. Nucleic Acids Res. 2017, 46, 1021-1037. [CrossRef]

6. Sau, S.P.; Kumar, P.; Sharma, P.K.; Hrdlicka, P.J. Fluorescent intercalator displacement replacement (FIDR) assay: Determination of relative thermodynamic and kinetic parameters in triplex formation-A case study using triplex-forming LNAs. Nucleic Acids Res. 2012, 40, e162. [CrossRef]

7. Liquier, J.; Gouyette, C.; Huynh-Dinh, T.; Taillandier, E. Raman spectroscopy of nucleic acid triple helices. J. Raman Spectrosc. 1999, 30, 657-666. [CrossRef]

8. Klener, J.; Štěpánek, J. UV resonance Raman study of PolyA and PolyU complexes: Mg2+-induced formation of PolyU.PolyA.PolyU triplexes. Vib. Spectrosc. 2015, 81, 32-39. [CrossRef]

9. Gfrörer, A.; Schnetter, M.E.; Wolfrum, J.; Greulich, K.O. Double and triple helices of nucleic acid polymers, studied by UVresonance Raman spectroscopy. Ber. Bunsenges. Phys. Chem. 1993, 97, 155-162. [CrossRef]

10. O'Connor, T.; Bina, M. The structure of triple helical Poly(U)·Poly(A)·Poly(U) studied by Raman spectroscopy. J. Biomol. Struct. Dyn. 1984, 2, 615-625. [CrossRef]

11. Schlücker, S. Surface-enhanced Raman spectroscopy: Concepts and chemical applications. Angew. Chem. Int. Edit. 2014, 53, 4756-4795. [CrossRef] [PubMed]

12. Zong, C.; Xu, M.X.; Xu, L.J.; Wei, T.; Ma, X.; Zheng, X.S.; Hu, R.; Ren, B. Surface-enhanced Raman spectroscopy for bioanalysis: Reliability and challenges. Chem. Rev. 2018, 118, 4946-4980. [CrossRef] [PubMed]

13. Wang, X.; Huang, S.-C.; Hu, S.; Yan, S.; Ren, B. Fundamental understanding and applications of plasmon-enhanced Raman spectroscopy. Nat. Rev. Phys. 2020, 2, 253-271. [CrossRef]

14. Garcia-Rico, E.; Alvarez-Puebla, R.A.; Guerrini, L. Direct surface-enhanced Raman scattering (SERS) spectroscopy of nucleic acids: From fundamental studies to real-life applications. Chem. Soc. Rev. 2018, 47, 4909-4923. [CrossRef]

15. Qi, G.; Wang, D.; Li, C.; Ma, K.; Zhang, Y.; Jin, Y. Plasmonic SERS Au nanosunflowers for sensitive and label-free diagnosis of DNA base damage in stimulus-induced cell apoptosis. Anal. Chem. 2020, 92, 11755-11762. [CrossRef]

16. Li, Y.; Gao, T.; Xu, G.; Xiang, X.; Han, X.; Zhao, B.; Guo, X. Base-Pair Contents and sequences of DNA double helices differentiated by surface-enhanced Raman spectroscopy. J. Phys. Chem. Lett. 2019, 10, 3013-3018. [CrossRef]

17. Li, Y.; Han, X.; Zhou, S.; Yan, Y.; Xiang, X.; Zhao, B.; Guo, X. Structural features of DNA G-quadruplexes revealed by surfaceenhanced Raman spectroscopy. J. Phys. Chem. Lett. 2018, 9, 3245-3252. [CrossRef] [PubMed]

18. Sagar, D.M.; Korshoj, L.E.; Hanson, K.B.; Chowdhury, P.P.; Otoupal, P.B.; Chatterjee, A.; Nagpal, P. High-throughput block optical DNA sequence identification. Small 2018, 14, 1703165. [CrossRef] [PubMed]

19. Morla-Folch, J.; Gisbert-Quilis, P.; Masetti, M.; Garcia-Rico, E.; Alvarez-Puebla, R.A.; Guerrini, L. Conformational SERS classification of K-Ras point mutations for cancer diagnostics. Angew. Chem. Int. Edit. 2017, 56, 2381-2385. [CrossRef] [PubMed]

20. Koo, K.M.; Wang, J.; Richards, R.S.; Farrell, A.; Yaxley, J.W.; Samaratunga, H.; Teloken, P.E.; Roberts, M.J.; Coughlin, G.D.; Lavin, M.F.; et al. Design and clinical verification of surface-enhanced Raman spectroscopy diagnostic technology for individual cancer risk prediction. ACS Nano 2018, 12, 8362-8371. [CrossRef] [PubMed]

21. Wang, J.; Koo, K.M.; Wee, E.J.H.; Wang, Y.; Trau, M. A nanoplasmonic label-free surface-enhanced Raman scattering strategy for non-invasive cancer genetic subtyping in patient samples. Nanoscale 2017, 9, 3496-3503. [CrossRef] [PubMed]

22. Dick, S.; Bell, S.E.J.; Alexander, K.J.; O'Neil, I.A.; Cosstick, R. SERS and SERRS detection of the DNA lesion 8-Nitroguanine: A self-labeling modification. Chem. Eur. J. 2017, 23, 10663-10669. [CrossRef] [PubMed]

23. Morla-Folch, J.; Alvarez-Puebla, R.A.; Guerrini, L. Direct quantification of DNA base composition by surface-enhanced Raman scattering spectroscopy. J. Phys. Chem. Lett. 2016, 7, 3037-3041. [CrossRef] [PubMed]

24. Morla-Folch, J.; Xie, H.-N.; Gisbert-Quilis, P.; Gómez-de Pedro, S.; Pazos-Perez, N.; Alvarez-Puebla, R.A.; Guerrini, L. Ultrasensitive direct quantification of nucleobase modifications in DNA by surface-enhanced Raman scattering: The case of cytosine. Angew. Chem. Int. Edit. 2015, 54, 13650-13654. [CrossRef] [PubMed]

25. Torres-Nunez, A.; Faulds, K.; Graham, D.; Alvarez-Puebla, R.A.; Guerrini, L. Silver colloids as plasmonic substrates for direct label-free surface-enhanced Raman scattering analysis of DNA. Analyst 2016, 141, 5170-5180. [CrossRef]

26. Fang, Y.; Bai, C.; Wei, Y.; Tang, Y. Surface-enhanced Fourier transform Raman scattering study on the adsorption structure of an RNA triple helix at a silver electrode. Appl. Spectrosc. 1996, 50, 48-52. [CrossRef]

27. Fang, Y.; Wei, Y.; Bai, C.; Kan, L.S. Surface-enhanced Fourier transform Raman scattering from a DNA triple helix poly[dA]·2poly[dT] at a silver electrode: Beyond the short-range mechanism. J. Phys. Chem. 1996, 100, 17410-17413. [CrossRef]

28. Sanchez-Cortes, S.; Garcia-Ramos, J.V. Anomalous Raman bands appearing in surface-enhanced Raman spectra. J. Raman Spectrosc. 1998, 29, 365-371. [CrossRef]

29. Guerrini, L.; Krpetić, Ž.; van Lierop, D.; Alvarez-Puebla, R.A.; Graham, D. Direct surface-enhanced Raman scattering analysis of DNA duplexes. Angew. Chem. Int. Edit. 2015, 54, 1144-1148. [CrossRef] 
30. Van Lierop, D.; Krpetic, Z.; Guerrini, L.; Larmour, I.A.; Dougan, J.A.; Faulds, K.; Graham, D. Positively charged silver nanoparticles and their effect on surface-enhanced Raman scattering of dye-labelled oligonucleotides. Chem. Commun. 2012, 48, 8192-8194. [CrossRef]

31. Kunkler, C.N.; Hulewicz, J.P.; Hickman, S.C.; Wang, M.C.; McCown, P.J.; Brown, J.A. Stability of an RNA•DNA-DNA triple helix depends on base triplet composition and length of the RNA third strand. Nucleic Acids Res. 2019, 47, 7213-7222. [CrossRef] [PubMed]

32. Gisbert-Quilis, P.; Masetti, M.; Morla-Folch, J.; Fitzgerald, J.M.; Pazos-Perez, N.; Garcia-Rico, E.; Giannini, V.; Alvarez-Puebla, R.A.; Guerrini, L. The structure of short and genomic DNA at the interparticle junctions of Cationic nanoparticles. Adv. Mater. Interfaces 2017, 4, 1700724. [CrossRef] 